DOE/ER/40853-5

\title{
RESEARCH IN ACCELERATOR PHYSICS (THEORY)
}

Final Technical Report

May 15, 1995 - August 14, 1996

Shoroku Ohnuma

Principal Investigator

The University of Houston

Houston, Texas 77204-5506

October 25, 1996

PREPARED FOR THE U.S.DEPARTMENT OF ENERGY UNDER GRANT NUMBER DE-FG03-94ER40853

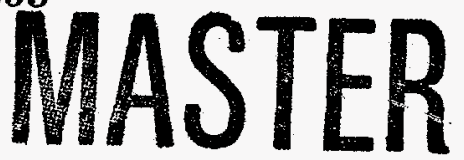




\section{DISCLAIMER}

Portions of this document may be illegible in electronic image products. Images are produced from the best available original document. 


\section{Introduction}

This is the final technical report of the grant DE-FG03-94ER40853 covering the period $5 / 15 / 94-8 / 14 / 96$, the final two years of the program in accelerator physics which started in August 1987.

During nine years of the project at the University of Houston, we have received $\$ 1,213,700$ from the Department of Energy under grants DE-FG05-87ER40374 and 94ER40853. In addition, we have been supported by two DOE laboratories, Fermilab and Argonne, with the total financial assistance of $\$ 110,050$. Thirteen post-doctoral research associates and eleven graduate students have been associated with the project, and five students graduated with doctoral degrees in physics. Three faculty members have received 13.5-month summer salaries and nine overseas trips have been made possible by the grants. Some 80 reports have been submitted to DOE as the result of our research activities.

Members of the group and their participation in the project during this grant period are:

S. Ohnuma (P.I.) Professor $\quad 35 \%$ (cost sharing) during academic year;

2.5 months in summer.

J. Shi Res. Asst. Prof. 5/15/94 - 12/31/94 100\%FTE

$1 / 01 / 95-5 / 14 / 9570 \%$ FTE

$5 / 15 / 95-2 / 29 / 96100 \% \mathrm{FTE}$

$3 / 01 / 96-6 / 14 / 9675 \% \mathrm{FTE}$

J. Liu Res. Asst. 6/01/94-7/31/94

\section{Research Activities}

During this grant period, research status and results have been reported in the following reports:

1. DOE/ER/40374-76

J. Shi, S. Ohnuma, and G. Wang, "Multi-parameter Sorting of Dipoles for Large Superconducting Rings", Proceedings of the Fourth European Particle Accelerator Conference, June 27 - July 1, 1994 (London), p. 1051.

2. DOE/ER/40374-77

Final Technical Report (May 15, 1993 - May 14, 1994). 


\section{DISCLAIMER}

This report was prepared as an account of work sponsored by an agency of the United States Government. Neither the United States Government nor any agency thereof, nor any of their employees, makes any warranty, express or implied, or assumes any legal liability or responsibility for the accuracy, completeness, or usefulness of any information, apparatus, product, or process disclosed, or represents that its use would not infringe privately owned rights. Reference herein to any specific commercial product, process, or service by trade name, trademark, manufacturer, or otherwise does not necessarily constitute or imply its endorsement, recommendation, or favoring by the United States Government or any agency thereof. The views and opinions of authors expressed herein do not necessarily state or reflect those of the United States Government or any agency thereof. 


\section{DOE/ER/40853-1}

"Integrable Polynomial Factorization for Symplectic Map Tracking in Large Storage Rings" in Accelerator Physics at the Superconducting Super Collider, edited by T. Yan, J. Naples, and M.J. Syphers, p. 678 (AIP, New York, 1995).

4. $\mathrm{DOE} / \mathrm{ER} / 40853-2$

"Transport Theory for Study of Tune Modulation" in Nonlinear Dynamics in Accelerator Physics, p. 184 (AIP, New York, 1995).

5. DOE/ER/40853-3

Technical Progress Report (May 15, 1994 - March 10, 1995).

6. DOE/ER/40853-4

"Chaotic Transport in Time-Dependent Symplectic Maps", J. Shi, Phys. Rev. Lett. 70 (1996), p. 3971.

7. "Sorting of Magnets in Large Superconducting Synchrotrons", J. Shi and S. Ohnuma, submitted to Phys. Rev. E., May 1996.

8. "Chaotic Transport and Particle Escape in Accelerators", J. Shi, submitted to Phys. Rev. E., Oct. 1996.

The last two papers will be submitted to the DOE after the publication.

During this project period, we have worked in the following three areas:

\section{Multi-Parameter Sorting of Dipoles for Large Superconducting Rings}

The presence of unavoidable multipole errors in superconducting magnets is known to be the main cause for limiting the dynamic aperture of large storage rings. Sorting of dipoles, in which dipoles are installed in the ring according to a certain sequence based on the measured multipole errors, is a way to reduce the adverse effects of random multipole errors without an increase in the cost. In order to reduce the effects of several multipole components simultaneously, we have developed a vector sorting scheme. In this sorting scheme, the multipole components are represented by a multi-dimensional vector and the sorting is based on the normal of the vector and the Euclidean distance between the vectors. During this project period, we have systematically studied the effectiveness of this sorting scheme with 
a test lattice which is similar to the high energy booster (HEB) of Superconducting Super Collider (SSC). The results of this study have been summarized in a paper which has been submitted to Phys. Rev. E.

a. Tracking studies of the dynamic aperture showed that the vector sorting scheme is effective in the improvement of the dynamic aperture even when more than one multipole components are responsible for the aperture limitation. When many multipoles are involved, an effective multi-parameter sorting needs to be done with a large number of dipoles in each sorting group. Figure 1 is a plot of the distribution of the dynamic aperture of fifty random samples with and without the sorting when all the multipole errors up to the 7 th order are included. It shows that a multi-parameter sorting with 232 dipoles in each sorting group is quite effective and the sorting effectively eliminates the particularly bad arrangements ("unlucky cases") as the spread of the dynamic aperture is significantly reduced after the sorting.

b. Linear coupling has been found to be an important factor to the reduction of dynamic aperture in some cases such as in LHC and Tevatron. Although the linear motion can be decoupled globally or even locally in most cases, a good dipole sorting scheme should be able to cope with the linear coupling. In order to examine the effectiveness of the vector sorting scheme in coupled systems, we have studied systems which is fully linearly coupled. Our study showed that the vector sorting scheme is effective in the enlargement of dynamic aperture in linearly coupled systems.

c. A strong nonlinearity in the lattice can lead to a substantial degree of amplitude dependence of betatron tunes even in a phase-space region near the origin, and this may result in crossings of dangerous resonances and a reduction in the dynamic aperture. Minimizing the amplitude dependence of tunes is thus desirable for a stable operation of the accelerator. we have investigated the effect of sorting on the reduction of the amplitude dependence of tunes due to the random error fields. Two methods, direct calculation of the average phase advance during the tracking and normal-form analysis of the one-turn map, have been employed for this purpose. The normal form analysis, which has been implemented in our code in an efficient way based on the Lie algebra formalism, provides a general and easy procedure to compute the amplitude dependence. The direct calculation of the average phase advance 
during tracking has been used to check the result of the normal-form analysis. Good agreement between these two methods has been found in our calculations. In Figs. 2 and 3, the shifts in tunes $\delta \nu_{x}$ and $\delta \nu_{y}$, averaged over fifty random samples, are plotted as a function of the action variables $I_{x}$ and $I_{y}$ when all the multipole errors up to the 7 th order are included. Without sorting, both horizontal and vertical tunes strongly depend on $I_{x}$ and $I_{y}$ as shown in Fig. 2. Since $\delta \vec{\nu}$ is calculated at one end of a long-straight section of the lattice where $\beta_{x}$ is much larger than $\beta_{y}\left(\beta_{x}=108.7 \mathrm{~m}\right.$ and $\left.\beta_{y}=19.1 \mathrm{~m}\right)$, this amplitude dependence is stronger in the horizontal motion than that in the vertical motion. Figure 3 plots the tune shifts of the lattice after the multi-parameter sorting of all the multipole components, where the sorting is performed with 232 dipoles in each sorting group. A comparison between the sorted and unsorted case shows that the sorting effectively suppresses the nonlinear tune shift. The linearity of the phase space is significantly improved after the sorting.

\section{Study of Tune Modulation}

Experiments in hadron accelerators have shown that the beam lifetime is substantially decreased when betatron tunes are modulated due to power supply ripples, ground motions, or synchro-betatron coupling. During last couple of years, we have developed the concept of chaotic transport in terms of flux across resonances to study the particle escape due to this tune modulation. It was shown that the transport theory provides a computationally efficient means of studying the particle escape and estimating the parameter dependence of the escape rate. During this project period, we have analysed the experimental results of the nonlinear beam experiments on the SPS of CERN by using the transport theory. In the SPS experiments, the particle loss rate was measured when the dominant resonance is the fifth-order resonance from sextuploes. We therefore studied the flux across the fifth-order resonance, which characterizes the particle loss rate. Many puzzling phenomena observed in the beam study experiments can be understood with the transport theory. The results of this studied has been published in Phys. Rev. Lett. A review of our study have been summarized in a paper which has been submitted to Phys. Rev. E.

a. The beam-particle loss due to the tune modulation has been studied on a ring with one saxtupole kick and otherwise linear. In order to examine the effect of the modulation 
when the modulation frequency $\omega$ is irrational, the flux across the fifth-order resonance when the modulation frequency approached irrational numbers with sequences of continuedfraction approximations. Table 1 lists the result of one example as $\omega$ approaches a goldenmean irrational number which is least easily approximable by rational numbers. When $\omega$ approaches the irrational number, the flux $\zeta$ converges very fast and no singularity was observed. Similar result was found for other irrational numbers. This result suggests that the effect of the tune modulation can be understood by only considering rational modulation frequencies, which significantly simplifies the problem.

Table 1: Effect of the modulation when the modulation frequency is an irrational number, $\omega=\frac{1}{350+\frac{1}{1+\ldots}}=$ $[350,1,1,1, \ldots] . \omega_{i}$ is the $i$ th-order continued-fraction approximation of $\omega . \nu_{0}=0.2114$.

\begin{tabular}{llcc}
\hline \hline & & \multicolumn{2}{c}{$\zeta\left(10^{-3}\right)$} \\
\cline { 3 - 4 }$\omega_{i}$ & $1 / \omega_{i}$ & $\left(\delta \nu=1 \times 10^{-3}\right)$ & $\left(\delta \nu=3 \times 10^{-3}\right)$ \\
\hline$[350]=\frac{1}{350}$ & 350 & 0.055582 & 0.50738 \\
{$[350,1]=\frac{1}{351}$} & 351 & 0.055542 & 0.50684 \\
{$[350,1,1]=\frac{2}{701}$} & 350.5 & 0.055562 & 0.50716 \\
{$[350,1,1,1]=\frac{3}{1052}$} & $350.666 \ldots$ & 0.055555 & 0.50706 \\
{$[350,1,1,1,1]=\frac{5}{1753}$} & 350.6 & 0.055558 & 0.50711 \\
\hline \hline
\end{tabular}

b. To understand the effect of higher-order resonances, we studied the flux across the 26thorder resonance which forms an island chain outside and very close to the 5 th-order resonance. Particles have to cross this island chain after crossing the 5th-order resonance in order to escape. The calculation of the flux across the 26 th-order resonance reveals a similar parameter dependence of the escape rate to that of the 5th-order resonance. This result suggests that the study on the 5th-order reseonance alone can provide the parameter dependence of the particle escape rate due to the tune modulation.

c. To examine the transport theory, a multi-particle tracking study has been conducted with an ensemble of $2 \times 10^{5}$ particles. Very good agreement was found between the result of the flux calculation and the result of the multi-particle tracking. 
d. It has been observed in the SPS experiments that the particle loss rate due to the tune modulation strongly depends on the modulation amplitude but weakly depends on the modulation frequency. This phenomenon had been a puzzle until an understanding based on our calculation. As it was observed in the experiments, our calculation showed that the effect of the tune modulation increases more than linearly with the modulation amplitude while is almost independent of the modulation frequency. In Table 2, a comparison is given between the amplitude dependence of the flux and the amplitude dependence of the particle loss rate measured in the SPS experiments. As the modulation amplitude $(\delta \nu)$ is increased from $1.1 \times 10^{-3}$ to $1.65 \times 10^{-3}$, both the flux and the loss rate are doubled. The particle loss due to the tune modulation is therefore well characterized by the flux when the modulation is weak. As $\delta \nu$ is further increased to $2.2 \times 10^{-3}$, the particle loss rate is increased by a factor of 10 while $\zeta$ is only increased by a factor of 4 . This discrepancy could be due to the additional particle escape along the vertical plane in the experiments when the modulation is strong. Even though flat beams were used (ratio of the vertical to horizontal beam size is 1:10) and the linear coupling was well corrected in the experiments, the particle escape along the vertical plane can be significant when the modulation is strong as it was observed in the experiments. The flux here has, however, only counted in the particle escape along the horizontal plane.

Table 2: A comparison of the $\delta \nu$ dependence of the particle loss rate measured in the SPS experiments and $\zeta . D_{1.1}$ and $\zeta_{1.1}$ are the values of the loss rate and $\zeta$ at $\delta \nu=1.1 \times 10^{-3}$, respectively.

\begin{tabular}{ccc}
\hline \hline$\delta\left(10^{-3}\right)$ & Loss rate & $\zeta$ \\
\hline 1.1 & $D_{1.1}$ & $\zeta_{1.1}$ \\
1.65 & $2 \times D_{1.1}$ & $2 \times \zeta_{1.1}$ \\
2.2 & $10 \times D_{1.1}$ & $4 \times \zeta_{1.1}$ \\
\hline \hline
\end{tabular}

\section{Integrable Polynomial Factorization for Symplectic Map Tracking}

Recently it has been shown that the integrable polynomial factorization is quite promising for the long-term tracking study. During this project period, we have made a computer code, 
based on the Lie algebra formalism, for constructing the integrable-polynomial-factorization map for the betatron motion in the transverse plane. Preliminary study of tracking up to five thousand turns on a test lattice which is similar to HEB of SSC has shown that tracking with the integrable-polynomial-factorization map and the element-by-element tracking give a very similar dynamic aperture. Tracking with a large number of turns and tracking with different "seed number", which is used to generate magnet arrangement, is currently underway.

\section{Other Activities}

S. Ohnuma spent four weeks each at Brookhaven and at Fermilab in the summer of 1995, and four weeks at Fermilab in the summer of 1996. He was also invited by the Japan Hadron Project (JHP) and spent four weeks at the Institute for Nuclear Study, University of Tokyo.

At Brookhaven, he worked on a simplified correction scheme for the linear horizontalvertical coupling in RHIC. An internal RHIC report was written describing the scheme. At Fermilab in 1995, he worked on the acceptance of the $\bar{p}$ accumulator, and in 1996, on the $\bar{p}$ recycler. In Japan, he investigated the impact of linear coupling on the required aperture of the high-intensity proton machine.

\section{Figure Caption}

Fig. 1. Dynamic aperture of fifty random samples when all the multipoles up to the 7th order are included. The number in each block identifies each sample. (a) dipoles are placed randomly; (b) all the multipole components are sorted with 32 dipoles in each sorting group; and (c) all the multipole components are sorted with 232 dipoles in each sorting group.

Fig. 2. $\delta \nu_{x}$ and $\delta \nu_{y}$, averaged over fifty random unsorted lattices; as functions of $I_{x}$ and $I_{y}$. All the multipoles up to the 7th order are included. The unit of $I_{x}$ and $I_{y}$ is $10^{-3} \mathrm{~mm}$.

Fig. 3. The average $\delta \nu_{x}$ and $\delta \nu_{y}$ of the fifty random samples as functions of $I_{x}$ and $I_{y}$. Conditions here are the same as those in Fig. 2 except that all multipole components are sorted with 232 dipoles in each sorting group. The unit of $I_{x}$ and $I_{y}$ is $10^{-3} \mathrm{~mm}$. 
Fig. I
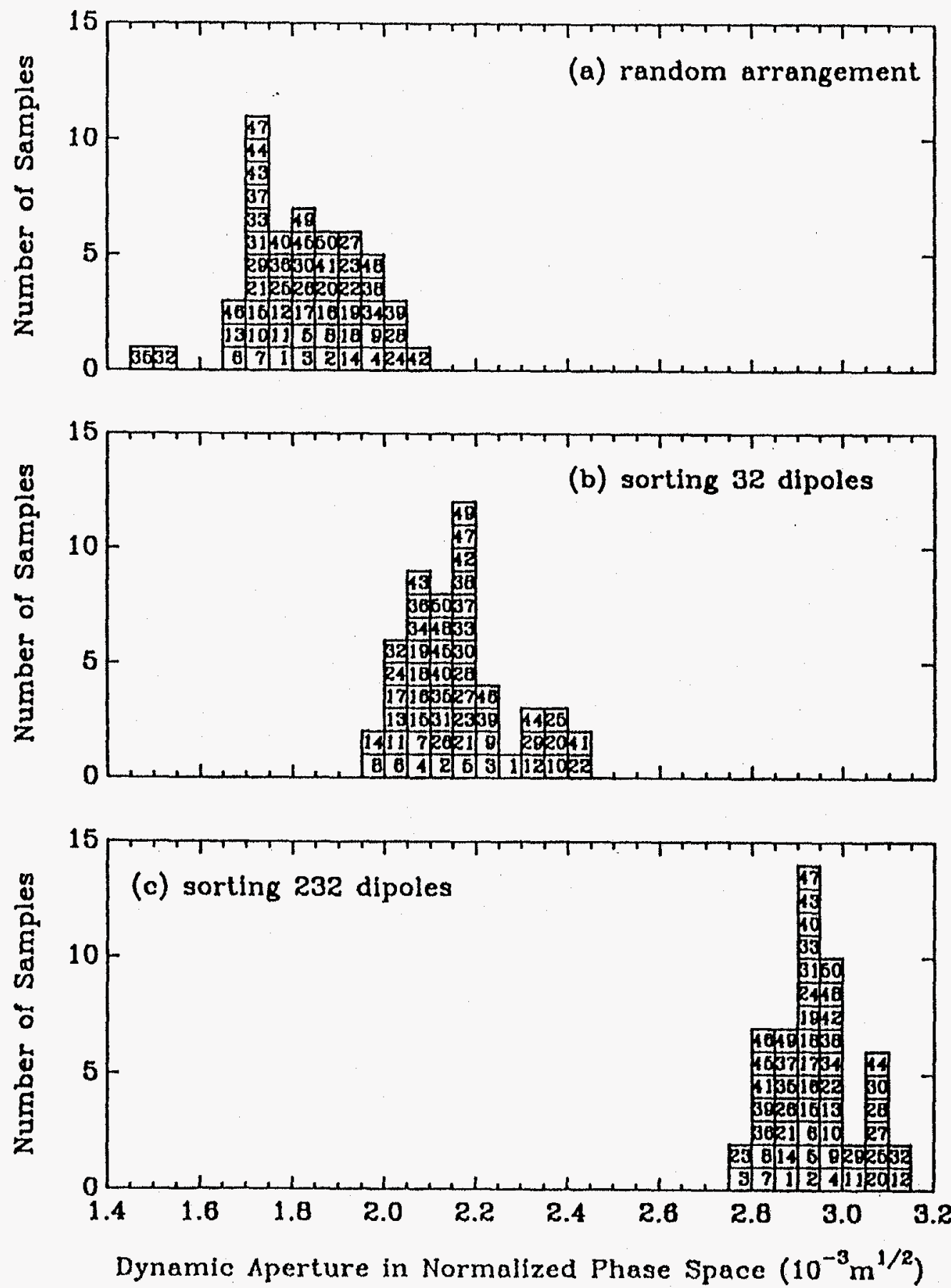


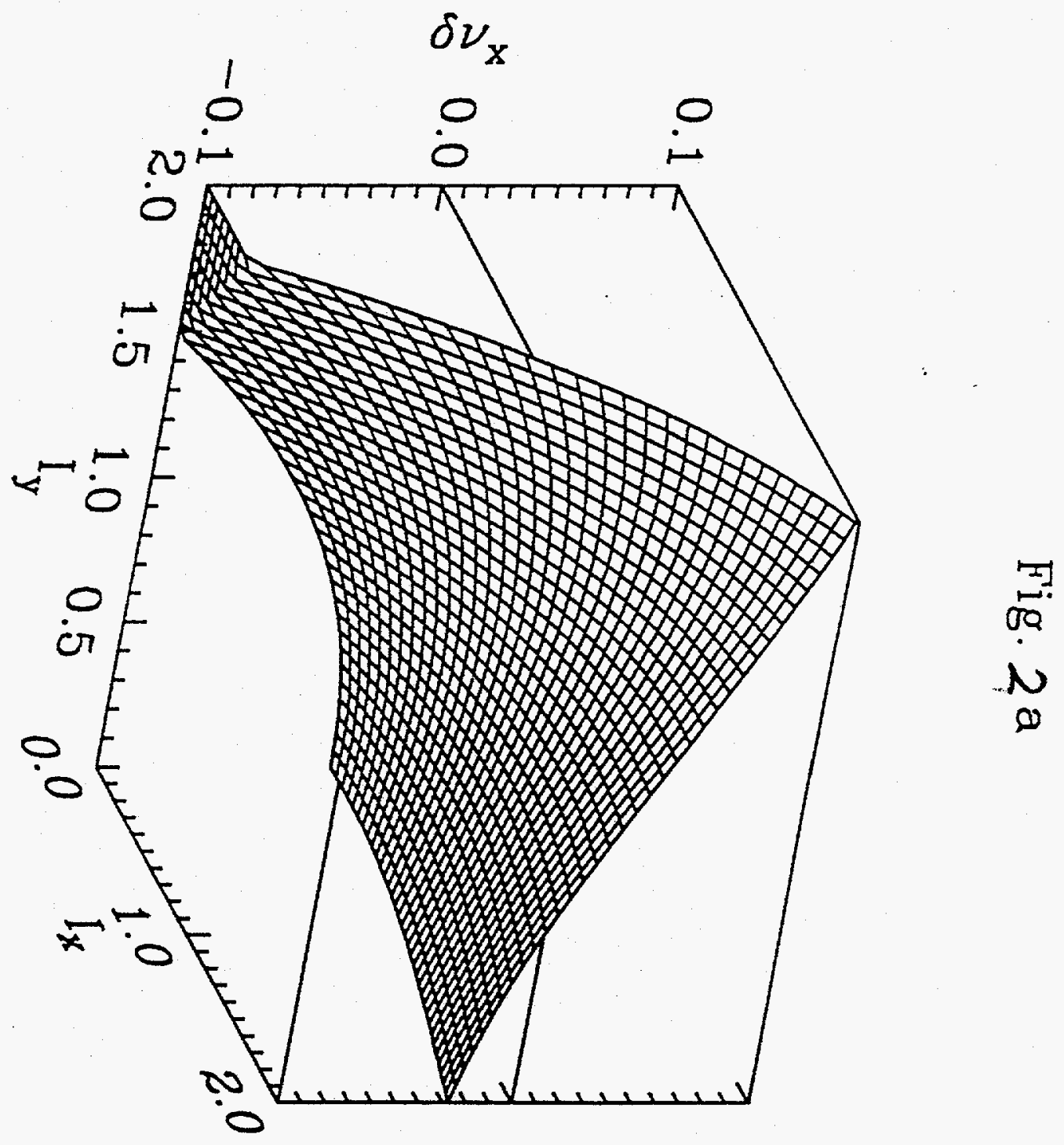




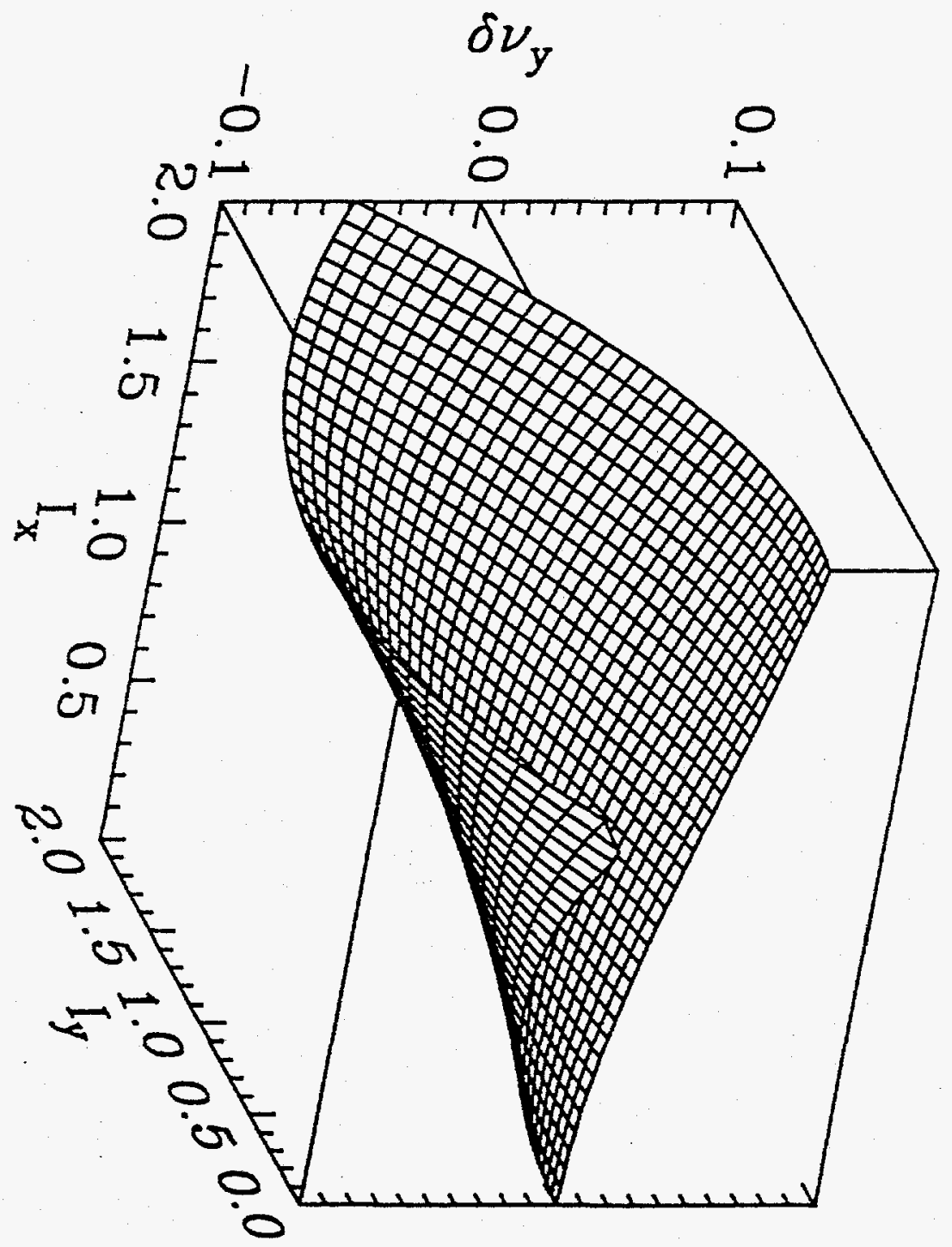

N 


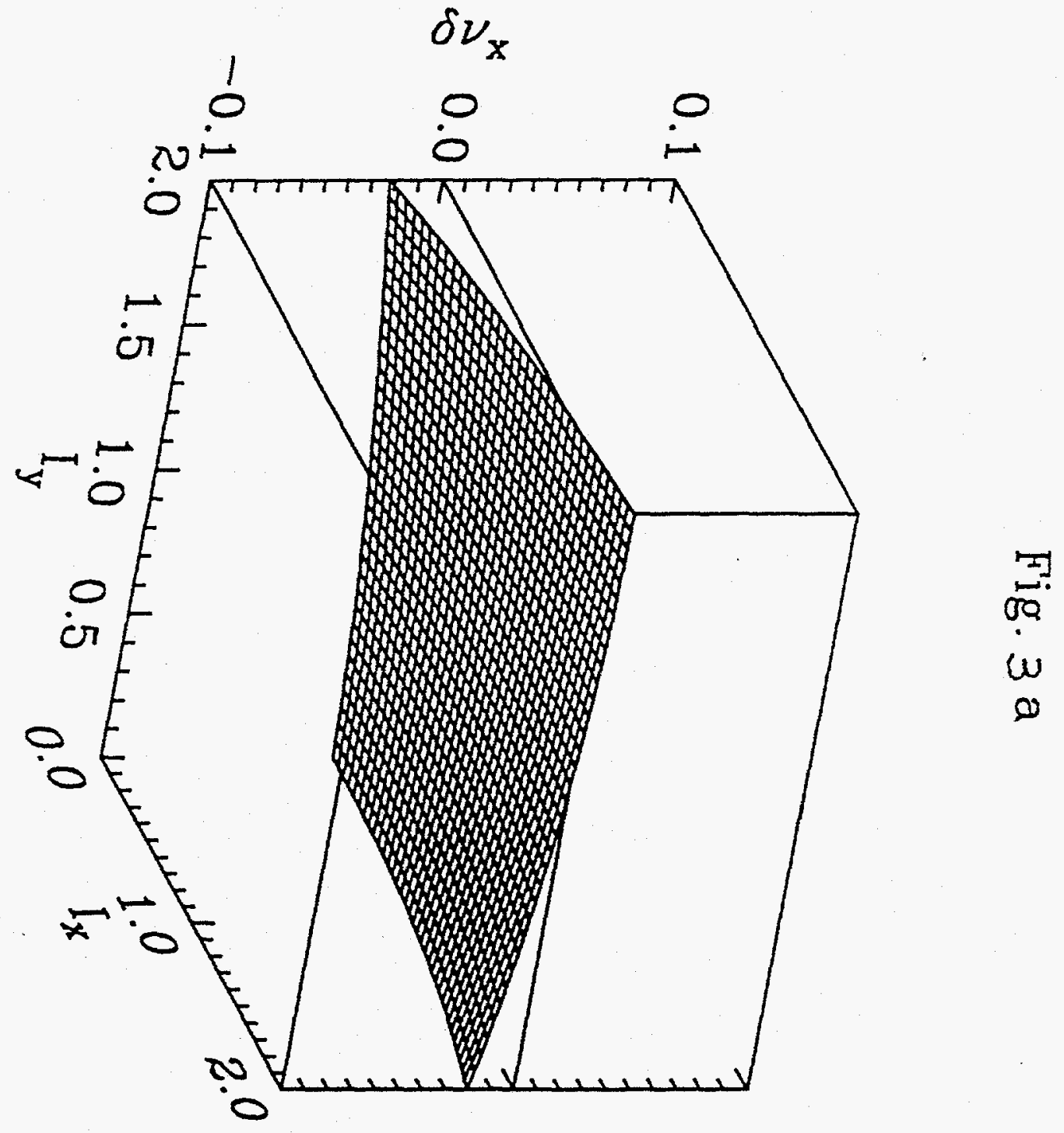




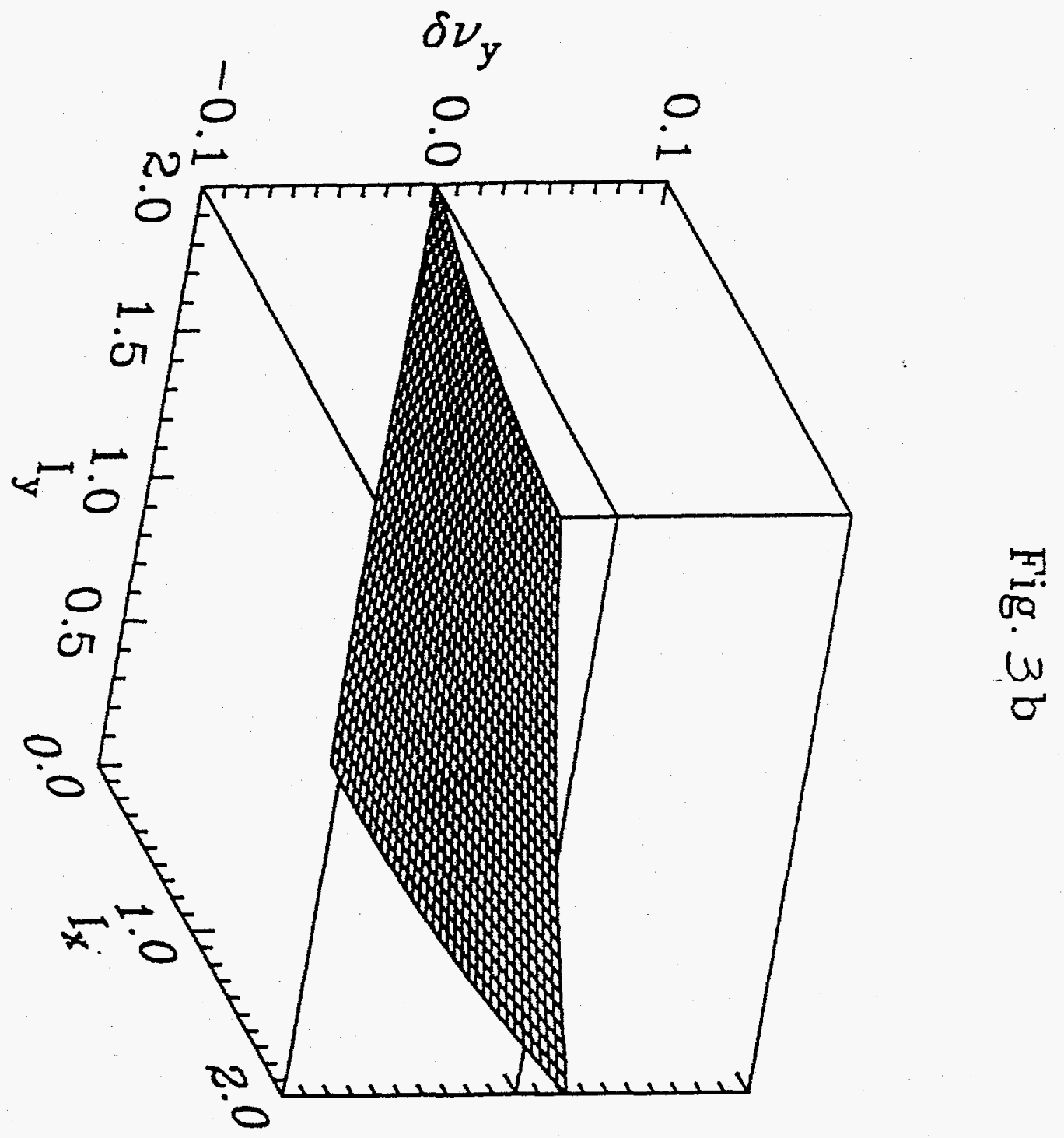

